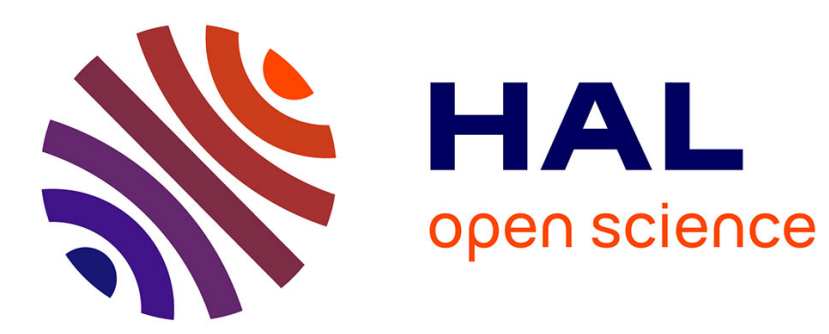

\title{
Algorithms for computing the Shapley value of cooperative games on lattices
}

Khaled Maafa, Lhouari Nourine, Mohammed Said Radjef

\section{To cite this version:}

Khaled Maafa, Lhouari Nourine, Mohammed Said Radjef. Algorithms for computing the Shapley value of cooperative games on lattices. 2018. hal-01702536

\section{HAL Id: hal-01702536 \\ https://hal.science/hal-01702536}

Preprint submitted on 6 Feb 2018

HAL is a multi-disciplinary open access archive for the deposit and dissemination of scientific research documents, whether they are published or not. The documents may come from teaching and research institutions in France or abroad, or from public or private research centers.
L'archive ouverte pluridisciplinaire HAL, est destinée au dépôt et à la diffusion de documents scientifiques de niveau recherche, publiés ou non, émanant des établissements d'enseignement et de recherche français ou étrangers, des laboratoires publics ou privés. 


\title{
Algorithms for computing the Shapley value of cooperative games on lattices.
}

\author{
Khaled MAAFA ${ }^{\mathrm{a}, \mathrm{b}}$, Lhouari NOURINE ${ }^{\mathrm{b}}$, Mohammed Said RADJEF ${ }^{\mathrm{a}}$ \\ ${ }^{a}$ Unité de Recherche LaMOS. Faculté des Sciences Exactes. Université de Bejaia, 06000 Bejaia, Algérie. \\ ${ }^{b}$ Université Clermont Auvergne, CNRS, LIMOS, France.
}

\begin{abstract}
We study algorithms to compute the Shapley value for a cooperative game on a lattice $\mathcal{L}_{\Sigma}=\left(\mathcal{F}_{\Sigma}, \subseteq\right)$ where $\mathcal{F}_{\Sigma}$ is the family of closed sets given by an implicational system $\Sigma$ on a set $N$ of players. The first algorithm is based on the generation of the maximal chains of the lattice $\mathcal{L}_{\Sigma}$ and computes the Shapley value in $O\left(|N|^{3} \cdot|\Sigma| .|C h|\right)$ time complexity using polynomial space, where $C h$ is the set of maximal chains of $\mathcal{L}_{\Sigma}$. The second algorithm proceeds by building the lattice $\mathcal{L}_{\Sigma}$ and computes the Shapley value in $O\left(|N|^{3}\right.$.| $\left.|\Sigma| .\left|\mathcal{F}_{\Sigma}\right|\right)$ time and space complexity. Our main contribution is to show that the Shapley value of weighted graph games on a product of chains with the same fixed length is computable in polynomial time. We do this by partitioning the set of feasible coalitions relevant to the computation of the Shapley value into equivalence classes in such a way that we need to consider only one element of each class in the computation.
\end{abstract}

Keywords: Cooperative game - Restricted cooperation - Lattice - Shapley value.

\section{Introduction}

The Shapley value [27] is one of the most popular solution concepts in the theory of cooperative games. It reflects the intuitive notion of fairness in the division of the output generated by a coalition of players. The Shapley value has been used in a wide range of applications such as clustering [14], congestion games [18] and network centrality [21]. Thus, the challenging task of computing the Shapley value has been one of the main concerns of researchers and practitioners in cooperative games. Most of the effort that has been prodigated to solve this issue deals with classical cooperative games i.e games where the set of feasible coalitions is the boolean lattice. In practice, however, not all the coalitions are feasible. For example, the feasible coalitions may be determined by linguistic or geographical factors. The solution concepts of classical cooperative games have to be

Email addresses: maafa.khaled@gmail.com (Khaled MAAFA), nourine@isima.fr (Lhouari NOURINE), radjefms@gmail.com (Mohammed Said RADJEF) 
redefined to take into account the restriction of the coalitional possibilities. Much effort has been done in this direction (see for example the survey in [16] and the references therein). In the framework of restricted cooperation, the Shapley value was defined for cooperative games with precedence constraints by Faigle and Kern [12], and for cooperative games on convex geometries by Bilbao and Edelman [3]. Recently, Faigle et al [11] have extended the definition of the Shapley value to cooperative games on concept lattices and therefore for general lattices.

The algorithmic aspects of solution concepts of games with restricted cooperation have not yet been sufficiently developed to permit their use in applications. The focus in this paper is on these algorithmic aspects. We address the issue of computing the Shapley value of a cooperative game on a lattice of sets closed under intersection, given by an implicational system $\Sigma$. From logic point of view, $\Sigma$ is a Horn CNF, and the models of $\Sigma$ are the characteristic vectors of the feasible coalitions $[2,30]$. The classical situation, where all the subsets of $N$ are feasible, occurs exactly when the implicational system is empty.

The complexity of computing the Shapley value of a game depends on how the input is given. Faigle and Kern [12] have shown that if the game is given by an oracle then the computation of the Shapley value is already exponential in the classical case. They have also shown that, for games with restricted cooperation, computing the Shapley value is \#P-hard even for elementary games given explicitly. Their reduction is based on counting the number of linear extensions of a poset, or minimal interval extensions when considering the concept lattice.

The paper is structured as follows. In Section 2, we relate our work to previous contributions and introduce the model of cooperative games on implicational systems. In Section 3, we define the problem of computing the Shapley value, decompose it into two sub-problems and discuss its difficulty. In Section 4, we give two algorithms for computing the Shapley value of cooperative games on implicational systems and discuss their complexity. In Section 5, we study the case where the lattice of feasible coalitions is isomorphic to a product of chains. We then show that when the chains are all of the same fixed length, we can compute in polynomial time the Shapley value of weighted graph games.

\section{Preliminaries and related work}

An implicational system on a set $N$ is a binary relation $\Sigma \subseteq 2^{N} \times N$. An implication $(A, i) \in \Sigma$ is denoted by $A \rightarrow i$.

Definition 1. Let $\Sigma=\left\{A_{1} \rightarrow a_{1}, \ldots, A_{m} \rightarrow a_{m}\right\}$ be an implicational system on $N$ and $X \subseteq N$. The $\Sigma$-closure of $X$, denoted $X^{\Sigma}$, is the smallest set containing $X$ and satisfying: $\forall 1 \leq j \leq m, A_{j} \subseteq X^{\Sigma} \Rightarrow a_{j} \in X^{\Sigma}$.

The set $\mathcal{F}_{\Sigma}=\left\{X^{\Sigma}, X \subseteq N\right\}$ is a closure system (closed under intersection and containing $N)$. The elements of $\mathcal{F}_{\Sigma}$ are referred to as $\Sigma$-closed sets. When ordered by inclusion, $\mathcal{F}_{\Sigma}$ is a lattice denoted by $\mathcal{L}_{\Sigma}=\left(\mathcal{F}_{\Sigma}, \subseteq\right)$. We will assume that $\emptyset \in \mathcal{F}_{\Sigma}$, that 
is, $\forall i \in N, \quad \rightarrow i \notin \Sigma$. Notice that we can remove from $\Sigma$ every implication $A \rightarrow i$ with $i \in A$, without changing the lattice of feasible coalitions. Therefore, we will consider only implications $A \rightarrow i$ with $i \notin A$. Figure 1 shows the lattice $\mathcal{L}_{\Sigma}$ corresponding to the implicational system $\Sigma=\{2 \rightarrow 1,4 \rightarrow 3,6 \rightarrow 5\}$ on $N=\{1, \ldots, 6\}$.

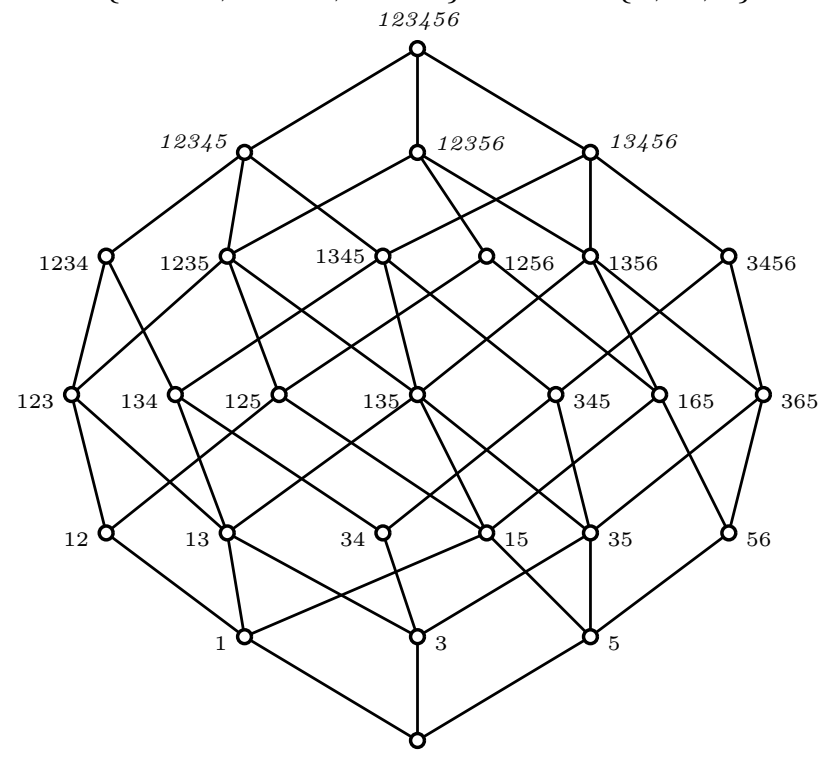

Figure 1: The lattice $\mathcal{L}_{\Sigma}$ corresponding to $\Sigma=\{2 \rightarrow 1,4 \rightarrow 3,6 \rightarrow 5\}$ on $N=\{1, \ldots, 6\}$.

We define a cooperative game on an implicational system as a game with restricted cooperation where the feasible coalitions are the closed sets of the given implicational system. More formally:

Definition 2. A cooperative game on an implicational system is a triplet $(N, \Sigma, v)$ where:

a) $N$ is a finite set of players;

b) $\Sigma$ is an implicational system on $N$;

c) $v$ is a mapping from $\mathcal{F}_{\Sigma}$ to $\mathbb{R}$ such that: $v(\emptyset)=0$.

The mapping $v$ assigns to every feasible coalition $F \in \mathcal{F}_{\Sigma}$ its worth, that is, the gain that the members of $F$ can achieve by themselves, regardless of how the players not in $F$ will act.

The notion of a conjuctive permission structure, introduced by Gilles et al [15] to model situations where a player may need the permission of another player to participate in the cooperative effort, can be translated to our framework, by restricting $\Sigma$ as follows:

$$
i \rightarrow j \in \Sigma \Leftrightarrow i \text { needs the permission of } j \text { to cooperate. }
$$


The autonomous coalitions in [15] will then be the $\Sigma$-closed sets in our framework. The idea of a conjunctive permission structure can even be generalized in our framework to include the case where a subset $A$ of players needs the presence of a player $i$ in order to put its cooperative possibilities into action. The player $i$ acts then as a catalyst to the cooperation of the players in $A$. This is simply achieved by setting $A \rightarrow i \in \Sigma$. Note however the difference between games with a permission structure as defined in [15] and our games on implicational systems which are games with restricted cooperation in the sense that only feasible coalitions can form. In [15] all coalitions can form but only their feasible part is able to generate worth. See [12] for more details on this point.

Many structural properties of the lattice $\mathcal{L}_{\Sigma}$ can be deduced directly from the form of the implicational system $\Sigma$. For instance we have the following proposition

Proposition 1. If $|A|=1$ for all $(A \rightarrow i) \in \Sigma$, then $\mathcal{L}_{\Sigma}$ is a distributive lattice.

Proof: Assume that $|A|=1$ for all $(A \rightarrow i) \in \Sigma$, then $\Sigma$ induces a permission structure $S$ on $N$, defined by $S(i)=\{j \in N \mid(j \rightarrow i) \in \Sigma\}$. $\mathcal{L}_{\Sigma}$ is then the collection of autonomous coalitions derived by the conjunctive approach from the permission structure $S$, and hence, is a distributive lattice. (See Definition 2.1 and Proposition 2.2 in [9]).

Note that the converse statement is not valid as shown in the following example:

$$
N=\{1,2,3,4,5\}, \quad \Sigma=\{1 \rightarrow 2, \quad 3 \rightarrow 4, \quad 5 \rightarrow 2, \quad 5 \rightarrow 4, \quad 24 \rightarrow 5\} .
$$

The lattice $\mathcal{L}_{\Sigma}$, depicted in figure 2 , is distributive despite the fact that $24 \rightarrow 5 \in \Sigma$

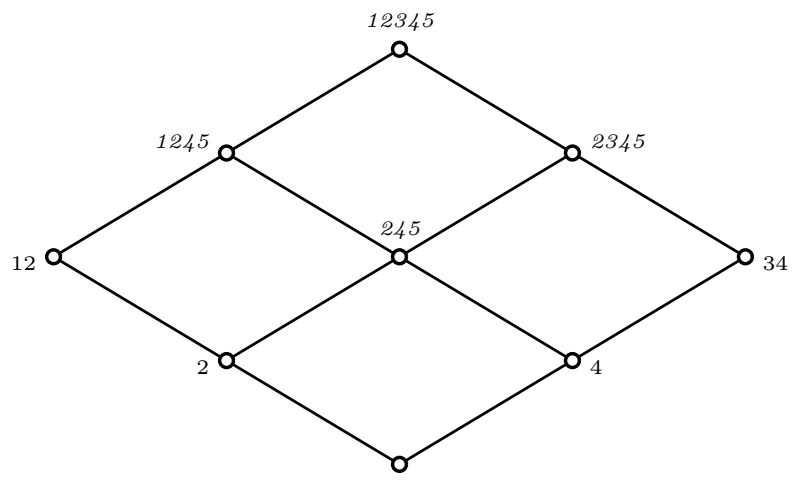

Figure 2: The lattice $\mathcal{L}_{\Sigma}$ corresponding to $\Sigma=\{1 \rightarrow 2,3 \rightarrow 4,5 \rightarrow 2, \quad 5 \rightarrow 4,24 \rightarrow 5\}$ on $N=\{1, \ldots, 5\}$.

Our work goes in the same line as those of Faigle and Kern [12], Bilbao and Edelman [3] and Faigle et al [11]. Cooperative games with precedence constraints, introduced in [12], correspond in our framework to the case $|A|=1$ for all $(A \rightarrow i) \in \Sigma$. We consider cooperative games on closure systems as in [11], with the difference that the feasible coalitions in our model are given by an implicational system $\Sigma$ on a set of players $N$, instead of a 
context as in [11]. We can shift from our representation to the representation of [11], in the following way:

Let $\Sigma$ be an implicational system. Consider the context $K=(G, M, I)$ defined by $G=N$, $M=\left\{F \in \mathcal{F}_{\Sigma} \mid \forall F_{1}, F_{2} \in \mathcal{F}_{\Sigma}, F \neq F_{1} \cap F_{2}\right\}$ and $I=\{(i, F) \in G \times M \mid i \in F\}$. The concept lattice of $K$ is isomorphic to $\mathcal{L}_{\Sigma}$. It is worth noticing, that these two representations are incomparable. Indeed, the size of an implicational system $\Sigma$ can be exponential in the size of the associated context and vice-versa. For example, if we define the feasible coalitions to be the cliques of a graph $G=(N, E)$ then we need $|N|^{2}$ implications in our representation, whereas the size of a representation by a context is exponential. On the other side one may need an exponential number of implications to represent the concepts of a context of polynomial size [20]. It might be noteworthy that sometimes the exponential blow-up can be avoided if auxiliary elements in $\mathrm{N}$ are allowed, as shown in [25].

\section{Position of the problem}

Let $(N, \Sigma, v)$ be a cooperative game on an implicational system. Let $C h$ be the set of maximal chains of the lattice $\mathcal{L}_{\Sigma}=\left(\mathcal{F}_{\Sigma}, \subseteq\right)$. For a maximal chain $c \in C h$ and $i \in N$, we denote by $F(c, i)$ the last coalition in $c$ that doesn't contain the player $i$, and by $F^{+}(c, i)$ the first coalition in $c$ that contains the player $i$.

The coalition $F^{+}(c, i)$ always covers the coalition $F(c, i)$ in the lattice $\mathcal{L}_{\Sigma}$, that is, there is no coalition $F$ in $\mathcal{L}_{\Sigma}$ such that $F(c, i) \subsetneq F \subsetneq F^{+}(c, i)$.

The Shapley value of $(N, \Sigma, v)$, as defined in [11], is the vector $\varphi(v)=\left(\varphi_{i}(v)\right)_{i \in N}$, where

$$
\varphi_{i}(v)=\frac{1}{|C h|} \sum_{c \in C h} \frac{v\left(F^{+}(c, i)\right)-v(F(c, i))}{\left|F^{+}(c, i) \backslash F(c, i)\right|} .
$$

In this paper, we consider the following problem:

\section{Shapley value}

Input: A cooperative game $(N, \Sigma, v)$.

Output: $\varphi(v)$.

Define the set

$$
\mathcal{A}_{i}=\left\{\left(F, F^{\prime}\right) \in \mathcal{F}_{\Sigma}^{2} \mid \exists c \in C h: F=F(c, i) \text { and } F^{\prime}=F^{+}(c, i)\right\} .
$$

Figure 3 shows the set $\mathcal{A}_{5}$ for $\Sigma=\{2 \rightarrow 1,4 \rightarrow 3,6 \rightarrow 5\}$ on $N=\{1, \ldots, 6\}$. For a maximal chain $c$, the coalition $F(c, 5)$ is linked to the coalition $F^{+}(c, 5)$ by a dotted line. The elements of $\mathcal{A}_{5}$ can be identified with the dotted lines. 


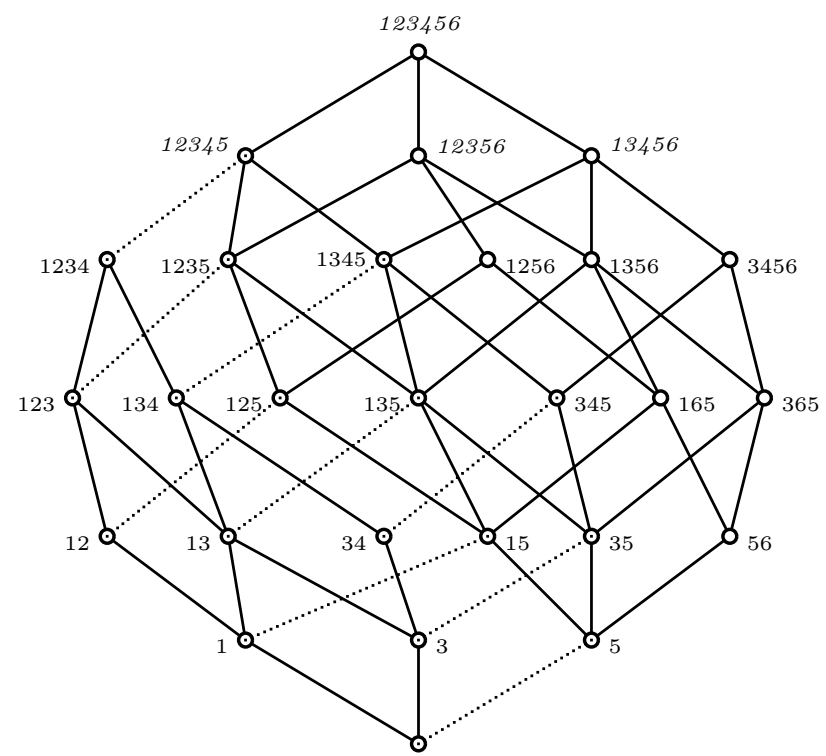

Figure 3: The dotted lines correspond to the elements of $\mathcal{A}_{5}$.

For any $F \in \mathcal{F}_{\Sigma}$, we denote by $C h^{\downarrow}(F)$ (resp. $C h^{\uparrow}(F)$ ) the number of maximal chains of the sublattice $[\emptyset, F]$ (resp. $[F, N])$. In Figure 3, we have $C h^{\downarrow}(135)=6$ and $C h^{\uparrow}(135)=6$. With this notation, equation (1) becomes

$$
\varphi_{i}(v)=\frac{1}{C h^{\downarrow}(N)} \sum_{\left(F, F^{\prime}\right) \in \mathcal{A}_{i}} \frac{C h^{\downarrow}(F) \cdot C h^{\uparrow}\left(F^{\prime}\right)}{\left|F^{\prime} \backslash F\right|}\left(v\left(F^{\prime}\right)-v(F)\right) .
$$

In view of the last expression, we can decompose the problem Shapley value into two sub-problems, MarginSets and NChains:

\section{MarginSets}

Input: An implicational system $\Sigma$ on the set of players $N$ and $i \in N$.

Output: Enumerate the set $\mathcal{A}_{i}$.

\section{NChains}

Input: An implicational system $\Sigma$ on the set of players $N$ and $F \in \mathcal{F}_{\Sigma}$.

Output: $C h^{\downarrow}(F)$.

There exist output polynomial time algorithms to enumerate the set $\mathcal{A}_{i}$ for any given implicational system $\Sigma[4,19,23]$, but the size of $\mathcal{A}_{i}$ may be exponential e.g. for $\Sigma=\emptyset$, we have $\left|\mathcal{A}_{i}\right|=2^{|N|-1}$.

For general cooperative games, when the function $v$ is given by an oracle, any algorithm that solves Shapley value will list all the pairs in $\mathcal{A}_{i}$. For particular games, one can obtain a partition of the set $\mathcal{A}_{i}$ of polynomial size, where the elements of each equivalence class have the same behaviour. This idea will be developed in Section 5. 
The problem NChains consists in counting the number of maximal chains in the lattice associated to the implicational system $\Sigma_{F}=\{X \rightarrow x \mid X \cup\{x\} \subseteq F\}$ on the set of players $F$. Notice that $C h^{\uparrow}(F)$ is obtained by solving the problem NChains with the arguments $F=N$ and $\Sigma^{\prime}=\Sigma \cup\{\emptyset \rightarrow j \mid j \in F\}$. Brightwell and Winkler [5] have shown that the problem NChains is \#P-Hard even for closure systems closed under union, i.e. distributive lattices.

The next proposition is a rewriting of results in [12] we therefore omit its proof.

Proposition 2. [12] Assume that we have an oracle solving NChains. Then an algorithm that solves Shapley value will execute a call for the oracle for each $\left(F, F^{\prime}\right) \in \mathcal{A}_{i}, i \in N$.

Thus, solving Shapley value is at least as hard as solving NChains. In other words, the complexity of NChains is a lower bound for the complexity of Shapley value even for elementary games.

In order to solve efficiently the problem Shapley value, we must be able to solve the problem NChains efficiently. This is, however, not sufficient. It may happen that we can solve NChains efficiently and yet the problem Shapley value remains hard. This is due to the interaction between the two sub-problems NChains and MarginSets.

In the next section, we give two algorithms for solving the problem Shapley value. The first one enumerates the maximal chains of $\mathcal{L}_{\Sigma}$. The second one computes the lattice $\mathcal{L}_{\Sigma}$, and then computes the Shapley value. In Section 5, we address the case where the lattice $\mathcal{L}_{\Sigma}$ is isomorphic to a product of chains.

\section{Two algorithms for computing the Shapley value}

In the following, we describe two algorithms for computing the Shapley value for any cooperative game $(N, \Sigma, v)$.

\subsection{Enumeration of the maximal chains of $\mathcal{L}_{\Sigma}$}

We will generate the maximal chains of the lattice $\mathcal{L}_{\Sigma}$ in a classical backtracking manner, i.e. given a partial chain $C=\left(\emptyset=F_{0}, \ldots, F_{j}\right)$, for each feasible set $F$ covering $F_{j}$ we will enumerate all the maximal chains having $\left(\emptyset=F_{0}, \ldots, F_{j}, F\right)$ as a prefix, starting from $C=(\emptyset)$. Whenever $F=N$, then a maximal chain is obtained and thus we compute the marginal contribution of each player in $N$ with respect to this maximal chain.

For a family $\mathcal{E}$ of sets, We denote by $\operatorname{Min}_{\subseteq}(\mathcal{E})$ the set of minimal elements of $\mathcal{E}$ with respect to set inclusion. Lemma 1 shows how to compute a feasible coalition covering another in the lattice $\mathcal{L}_{\Sigma}$. This lemma is not new, it can be found in earlier papers dealing with the construction of the Galois or concept lattice of a context. See for example [4]. Our contribution is to show how to compute a covering coalition using $\Sigma$ as an input.

Lemma 1. $F^{\prime}$ covers $F$ in $\mathcal{L}_{\Sigma}$ iff $F^{\prime} \in M i n_{\subseteq}\left\{(F \cup\{i\})^{\Sigma}, i \in N \backslash F\right\}$. 
Proof: Suppose $F^{\prime}$ covers $F$ and let $j \in F^{\prime} \backslash F$. Then $F \subset(F \cup\{j\})^{\Sigma}=F^{\prime}$ otherwise $F^{\prime}$ does not cover $F$. Now suppose there exists $i \in N \backslash F$ such that $F \subset(F \cup\{i\})^{\Sigma} \subset(F \cup\{j\})^{\Sigma}=F^{\prime}$. This contradicts the fact that $F^{\prime}$ covers $F$, and thus $F^{\prime} \in \operatorname{Min}_{\subseteq}\left\{(F \cup\{i\})^{\Sigma}, i \in N \backslash F\right\}$.

Conversely suppose $F^{\prime} \in M i n \subseteq\left\{(F \cup\{i\})^{\Sigma}, i \in N \backslash F\right\}$, with $F^{\prime}=(F \cup\{j\})^{\Sigma}, j \in N \backslash F$ and $F^{\prime}$ does not cover $F$. Let $F \subset F^{\prime \prime} \subset F^{\prime}$ and $i \in F^{\prime \prime} \backslash F$. Then $F \subset F^{\prime \prime}=(F \cup\{i\})^{\Sigma} \subset$ $F^{\prime}=(F \cup\{j\})^{\Sigma}$ which contradicts the fact that $(F \cup\{j\})^{\Sigma}$ is minimal.
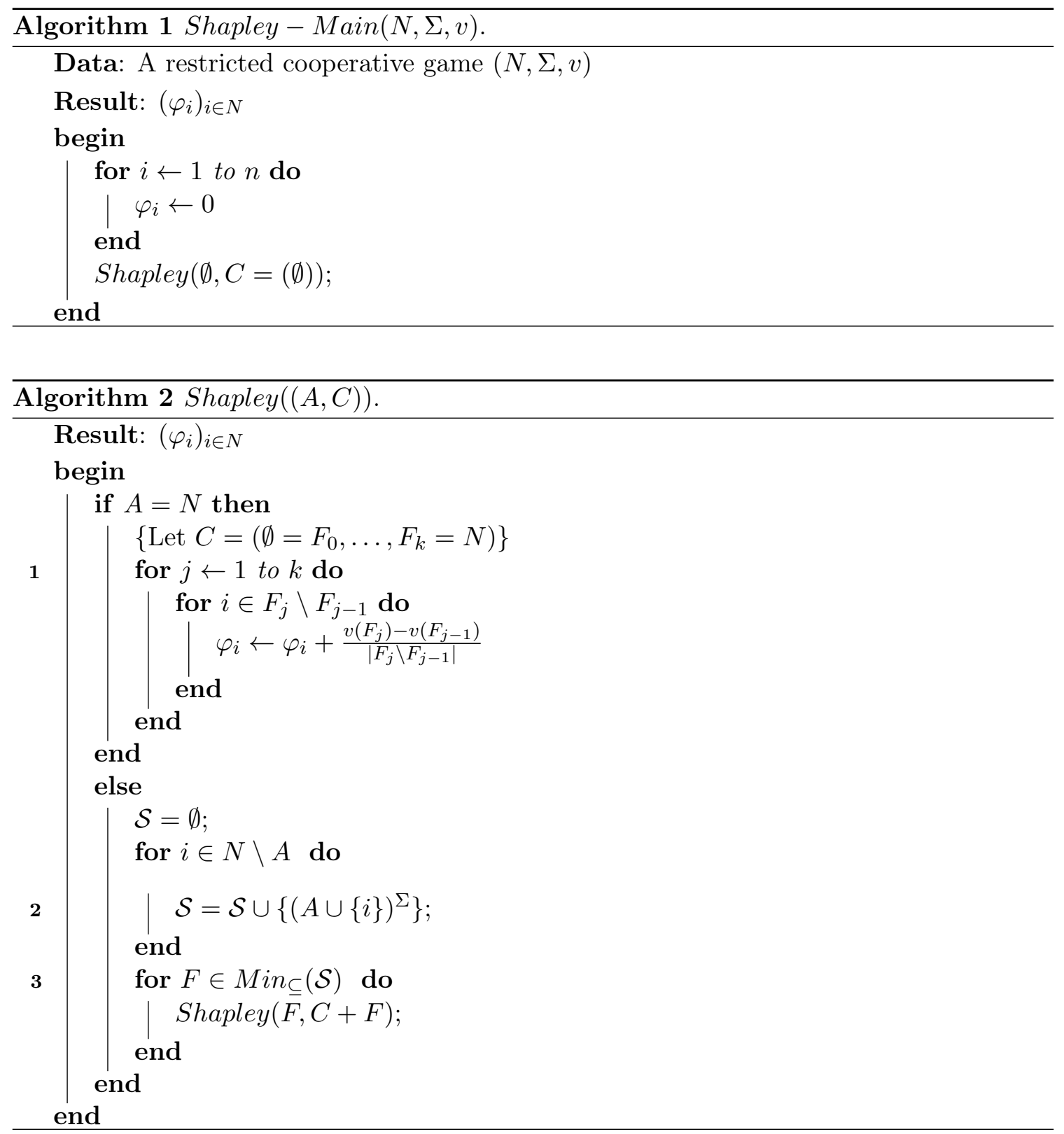
Proposition 3. Algorithm 1 computes the Shapley value in $O\left(|N|^{3} \cdot|\Sigma| \cdot \mid\right.$ Ch|) time complexity using polynomial space.

Proof: Algorithm 1 enumerates maximal chains recursively, and at each node $A$ of the execution tree, it computes the feasible coalitions covering $A$ in the lattice using Lemma 1. For each leaf, the algorithm computes the marginal contributions associated to the corresponding maximal chain (see Line 1 of Algorithm 2).

The complexity of Algorithm 2 is strongly related to the complexity of Line 2. Wild [29] gives an algorithm for computing the closure of a set using an implicational system in $O\left(|N|^{2} \cdot|\Sigma|\right)$. Thus, the complexity for outputting a maximal chain is bounded by $O\left(|N|^{3} \cdot|\Sigma|\right)$, since the length of a chain is at most $|N|$.

\subsection{Building the lattice $\mathcal{L}_{\Sigma}$}

The strategy here is first to compute the feasible coalitions lattice, and then compute the Shapley value. There are several algorithms for computing the lattice $[1,4,23]$ that are already implemented in several platforms (e.g. Galicia or concepts explorer).

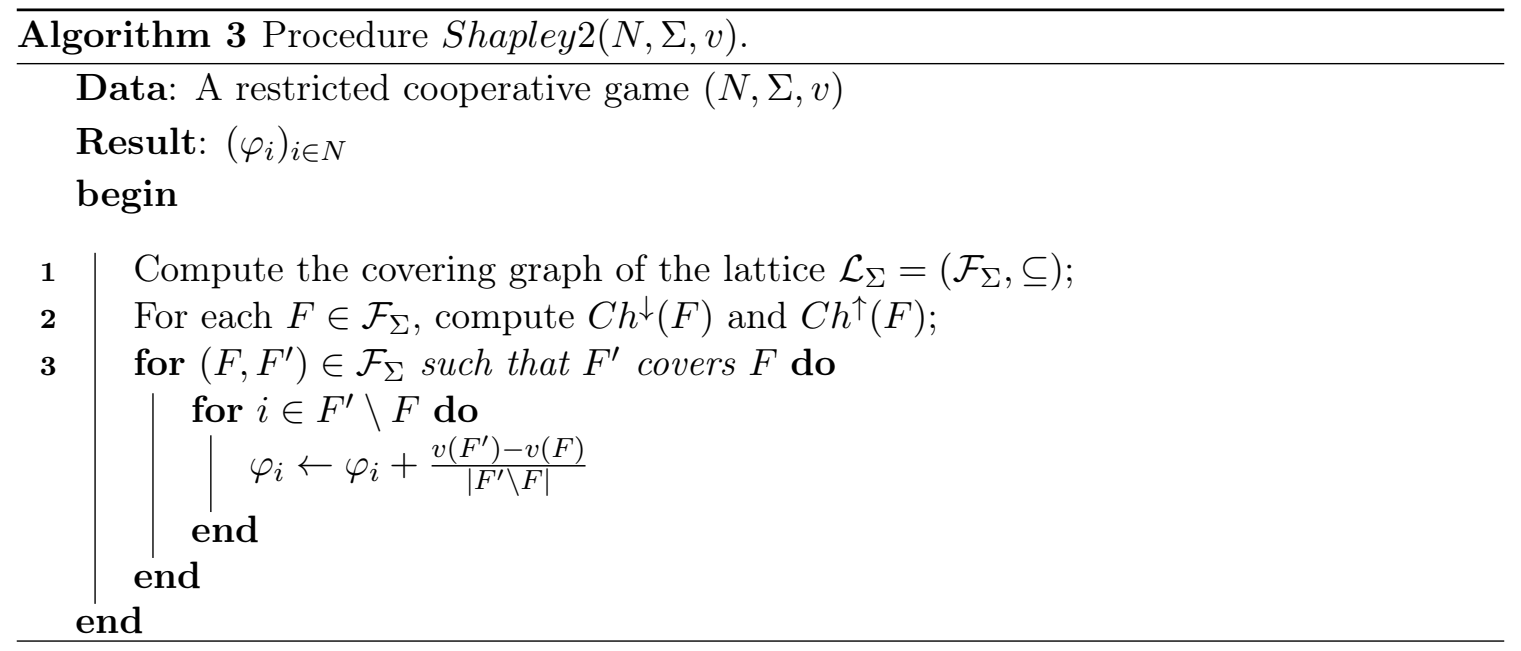

Proposition 4. Algorithm 3 computes the Shapley value in $O\left(|N|^{3} \cdot|\Sigma| .\left|\mathcal{F}_{\Sigma}\right|\right)$ time and space complexity.

Proof: The complexity of the computation of the lattice based on an implicational system can be easily derived from the complexity of algorithms in $[1,4,23]$ and can be bounded by $O\left(|N|^{3} \cdot|\Sigma| .\left|\mathcal{F}_{\Sigma}\right|\right)$. The computation of $C h^{\downarrow}(F)$ for every $F \in \mathcal{F}_{\Sigma}$ can be done in $O\left(|N| .\left|\mathcal{F}_{\Sigma}\right|\right)$ using a breadth first search (BFS) of the covering graph of the lattice $\mathcal{L}_{\Sigma}$ starting from the bottom. The same argument applies to computing $C h^{\uparrow}(F)$ starting from the top of the lattice. The line 3 is simple search of the covering graph of the lattice $\mathcal{L}_{\Sigma}$, and for each 
edge the computation time is constant. Thus, the total complexity is bounded by the computation of the lattice (line 1).

Notice that whenever the time complexity of Algorithm 1 is polynomial then Algorithm 3 is also polynomial, since $|C h| \geq \frac{\left|\mathcal{F}_{\Sigma}\right|}{N}$.

\section{Weighted graph games on a product of chains}

In this section, we restrict our attention to implicational systems which yield a collection of feasible coalitions isomorphic to a product of chains. This situation occurs, for example, in the model of multi-choice games introduced by Hsiao and Raghavan [17] and further studied in [24]. The problem NChains can then be solved efficiently. The sets $\mathcal{A}_{i}, i \in N$, will be partitioned into equivalence classes in such a way that we need only to consider one element of each class in the computation of the Shapley value. If the number of classes is polynomial, the Shapley value may be computed in polynomial time for specific classes of games.

Let $C_{1}, \ldots, C_{m}$ be disjoint finite sets. Assume that for each $t$ in $\{1, \ldots, m\}, C_{t}$ is totally ordered by $\preccurlyeq_{t}$. We thus have $m$ disjoint totally ordered sets $\left(C_{1}, \preccurlyeq 1\right), \ldots\left(C_{m}, \preccurlyeq m\right)$. The size of a chain is defined as the number of its elements and the length of a chain is defined as its size minus one. Without loss of generality, we can assume that all the chains have the same size $l$, and hence, the same length $l-1$. See remark 2 at the end of the paper for the case where the chains are not of the same length.

Let $N=\bigcup_{t=1}^{m} C_{t}$ and $|N|=n$. We define the partial order $P=(N, \preccurlyeq)$, called the parallel sum of the chains $\left(C_{1}, \preccurlyeq 1\right), \ldots\left(C_{m} \preccurlyeq m\right)$, by

$$
\forall i, j \in N: \quad i \preccurlyeq j \Leftrightarrow \exists t \in\{1, \ldots, m\}: i \preccurlyeq t j .
$$

We associate with the partial order $P$ the implicational system $\Sigma$ on $N$ defined by

$$
\Sigma=\{i \rightarrow j \mid i, j \in N, \quad j \preccurlyeq i\} .
$$

An ideal of $P$ is a subset $I$ of $P$ such that $j \in I$ and $i \preccurlyeq j$ implies $i \in I$. Clearly $\mathcal{L}_{\Sigma}=\left(\mathcal{F}_{\Sigma}, \subseteq\right)$ is the lattice of ideals of $P$.

The product of the chains $\left(C_{1}, \preccurlyeq 1\right), \ldots\left(C_{m} \preccurlyeq m\right)$ is the partial order $\left(C_{1} \times C_{2} \times \cdots \times C_{m}, \preccurlyeq\right.$ prod $)$, where

$$
\left(x_{1}, x_{2}, \cdots, x_{m}\right) \preccurlyeq_{\text {prod }}\left(y_{1}, y_{2}, \cdots, y_{m}\right) \Leftrightarrow x_{t} \preccurlyeq_{t} y_{t} \forall t \in\{1,2, \cdots, m\} \text {. }
$$

To see that the lattice $\mathcal{L}_{\Sigma}=\left(\mathcal{F}_{\Sigma}, \subseteq\right)$ is isomorphic to a product of chains, observe that any $F \in \mathcal{L}_{\Sigma}$ is uniquely determined by the set

$$
\max (F)=\{i \in F \mid i \npreceq j \forall j \in F\} .
$$


Indeed, we have

$$
F=\{i \in N \mid \exists j \in \max (F): i \preccurlyeq j\} .
$$

$F \in \mathcal{L}_{\Sigma}$ can thus be identified with the $m$-tuple $\left(i_{1}, i_{2}, \cdots, i_{m}\right)$ where, for $t=1,2, \cdots m, i_{t}$ is the unique player in $\max (F) \cap C_{t}$ if $\max (F) \cap C_{t} \neq \emptyset$, and $i_{t}=r_{t}$ with $r_{t}$ is an imaginary player appended at the bottom of the chain $\left(C_{t}, \preccurlyeq_{t}\right)$, if $\max (F) \cap C_{t}=\emptyset$.

Letting $\left(C_{t}^{\prime}, \preccurlyeq_{t}\right)$ be the obtained chain after appending the player $r_{t}$ at the bottom of $\left(C_{t}, \preccurlyeq t\right.$ ), the lattice $\mathcal{L}_{\Sigma}=\left(\mathcal{F}_{\Sigma}, \subseteq\right)$ is isomorphic to the product of chains $\left(C_{1}^{\prime} \times C_{2}^{\prime} \times \cdots \times C_{m}^{\prime}, \preccurlyeq\right.$ prod $)$. This fact is illustrated in the following example.

Example 1. Consider the three chains $C_{1}: 1 \preccurlyeq_{1} 2, C_{2}: 3 \preccurlyeq_{2} 4, C_{3}: 5 \preccurlyeq_{3} 6$. The Hasse diagram of the partial order $P$ is depicted in figure $4 a$. In figure $4 b$, we have added an imaginary player at the bottom of each chain of $P$. The implicational system associated with $P$ is $\Sigma=\{2 \rightarrow 1,4 \rightarrow 3,6 \rightarrow 5\}$. We have $n=6, m=3, l=2$. The lattice $\mathcal{L}_{\Sigma}$ is depicted in figure $5 a$ and is isomorphic to the product of chains $\left(C_{1}^{\prime}, \preccurlyeq 1\right) \times\left(C_{2}^{\prime}, \preccurlyeq 2\right) \times\left(C_{3}^{\prime}, \preccurlyeq 3\right)$ depicted in figure $5 b$.

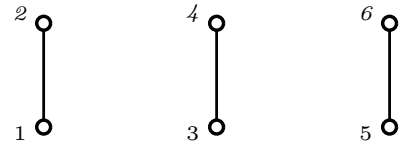

(a) The partial order $P$.

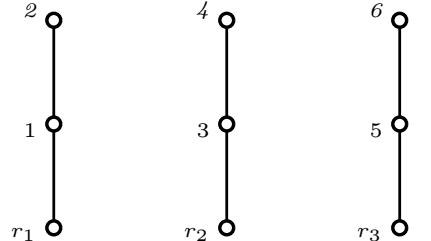

(b) The chains $\left(C_{t}^{\prime}, \preccurlyeq t\right), t=1,2,3$.

Figure 4: Adding imaginary players at the bottom of each chain of $P$. 


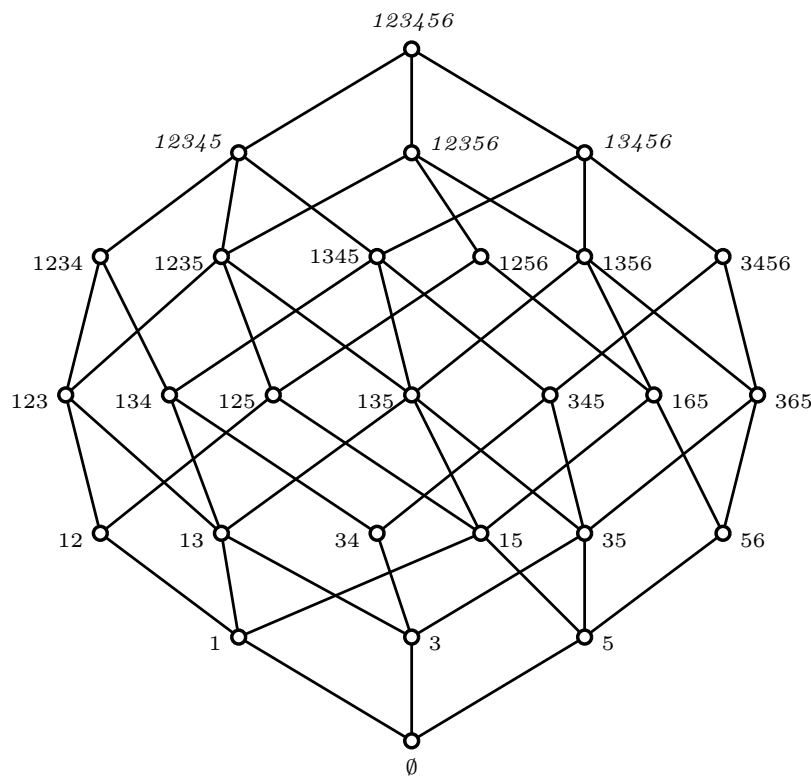

(a) The lattice $\mathcal{L}_{\Sigma}$.

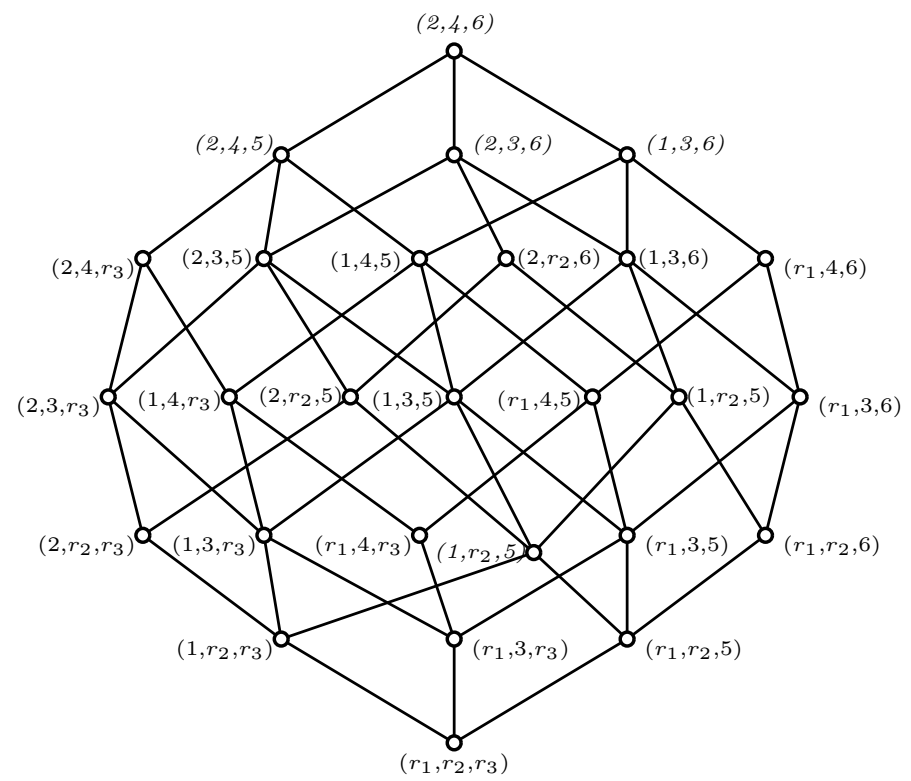

(b) $\left(C_{1}^{\prime}, \preccurlyeq_{1}\right) \times\left(C_{2}^{\prime}, \preccurlyeq_{2}\right) \times\left(C_{3}^{\prime}, \preccurlyeq 3\right)$.

Figure 5: The lattice $\mathcal{L}_{\Sigma}$ is isomorphic to the product $\left(C_{1}^{\prime}, \preccurlyeq_{1}\right) \times\left(C_{2}^{\prime}, \preccurlyeq 2\right) \times\left(C_{3}^{\prime}, \preccurlyeq 3\right)$.

For $F \in \mathcal{F}_{\Sigma}$, let $P_{\mid F}$ denote the restriction of the partial order $P$ to the elements of $F$. For $i \in N$, let $h(i)=\left|\{i\}^{\Sigma}\right|-1$, i.e. the number of players preceding $i$ in the order $P$.

The fact that $\mathcal{L}_{\Sigma}$ is the lattice of ideals of $P$ enables us to give an alternative formulation for the sets $\mathcal{A}_{i}, i \in N$, where

$$
\mathcal{A}_{i}=\left\{\left(F_{1}, F_{2}\right) \in \mathcal{F}_{\Sigma}^{2} \mid \exists c \in C h: F_{1}=F(c, i) \text { and } F_{2}=F^{+}(c, i)\right\} .
$$

Proposition 5. Let $i \in N$ and $\Sigma$ defined as in (3). Then the elements of $\mathcal{A}_{i}$ are exactly the pairs $\left(F \cup\{i\}^{\Sigma} \backslash\{i\}, F \cup\{i\}^{\Sigma}\right)$ where $F \in \mathcal{F}_{\Sigma}$ with $F \cap C(i)=\emptyset$.

Proof: As $\mathcal{L}_{\Sigma}$ is the lattice of ideals of the order $P$, we know [28, p 291] that the coalitions that cover a coalition $F \in \mathcal{L}_{\Sigma}$ are just the coalitions $F \cup\{j\}$ where $j$ is minimal in $P \backslash F$. Thus for $\left(F_{1}, F_{2}\right) \in \mathcal{A}_{i}$ we have $F_{2}=F_{1} \cup\{i\}$ since $F_{2}$ covers $F_{1}$ and $i \in F_{2} \backslash F_{1}$.

Since $F_{1}=F_{2} \backslash\{i\}$ is an ideal of $P$, we necessarily have $i \in \max \left(F_{2}\right)$. Thus $F_{2}=F \cup\{i\}^{\Sigma}$ where $F$ is an ideal of $P$, hence $F \in \mathcal{F}_{\Sigma}$, with $F \cap C(i)=\emptyset$. Consequently, $\left(F_{1}, F_{2}\right)=$ $\left(F \cup\{i\}^{\Sigma} \backslash\{i\}, F \cup\{i\}^{\Sigma}\right)$.

Conversely, if $\left(F_{1}, F_{2}\right)=\left(F \cup\{i\}^{\Sigma} \backslash\{i\}, F \cup\{i\}^{\Sigma}\right)$ with $F \in \mathcal{F}_{\Sigma}$ and $F \cap C(i)=\emptyset$ then $F_{2}$ covers $F_{1}$ since $i$ is minimal in $P \backslash F_{1}$. In addition $i \in F_{2} \backslash F_{1}$, then we can build a maximal chain $c$ in $\mathcal{L}_{\Sigma}$ such that $F_{1}=F(c, i)$ and $F_{2}=F^{+}(c, i)$. Hence $\left(F_{1}, F_{2}\right) \in \mathcal{A}_{i}$. 
In view of the last proposition, the elements $\left(F \cup\{i\}^{\Sigma} \backslash\{i\}, F \cup\{i\}^{\Sigma}\right) \in \mathcal{A}_{i}$ can be identified with those of the set:

$$
\tilde{\mathcal{A}}_{i}=\left\{F \in \mathcal{F}_{\Sigma} \mid F \cap C(i)=\emptyset\right\} .
$$

We define an equivalence relation $\mathcal{R}_{i}$ on $\tilde{\mathcal{A}}_{i}$ as follows:

$$
F_{1} \mathcal{R}_{i} F_{2} \Leftrightarrow P_{\mid F_{1}} \text { is isomorphic to } P_{\mid F_{2}} .
$$

Let $\mathcal{Q}_{i}$ be the quotient set of $\tilde{\mathcal{A}}_{i}$ modulo $\mathcal{R}_{i}$. We will denote the class of $F \in \tilde{\mathcal{A}}_{i}$ by $\bar{F}$. Observe that all the elements of an equivalence class modulo $\mathcal{R}_{i}$ have the same cardinal, since $P_{\mid F_{1}}$ isomorphic to $P_{\mid F_{2}}$ implies $\left|F_{1}\right|=\left|F_{2}\right|$, and that this common cardinal is an integer in $[0, n-l]$. The next proposition gives an encoding of the class $\bar{F}$, with $|F|=k$, by a vector of integers in the set:

$$
\mathcal{D}_{k}=\left\{\left(x_{0}, \ldots, x_{l}\right) \in \mathbb{N}^{l+1}, \text { such that } \sum_{t=0}^{l} x_{t}=m-1, \quad \sum_{t=0}^{l} t \cdot x_{t}=k\right\} .
$$

We recall that $m$ is the number of the disjoint chains in the order $P$ and $l$ is their common size. In the rest of this paper, we denote the set $\bigcup_{k=0}^{n-l} \mathcal{D}_{k}$ by $\mathcal{E}$.

Proposition 6. Let $i \in N$. The sets $\mathcal{Q}_{i}$ and $\mathcal{E}=\bigcup_{k=0}^{n-l} \mathcal{D}_{k}$ are in bijection by the mapping $\psi: \mathcal{Q}_{i} \rightarrow \mathcal{E}, \bar{F} \mapsto \psi(\bar{F})=\left(x_{0}, \ldots, x_{l}\right)$ where $x_{t}$ is the number of chains of size $t$ in $P_{\mid F}$ for $1 \leq t \leq l$, and $x_{0}=m-1-\sum_{t=1}^{l} x_{t}$.

Furthermore, we have $\psi(\bar{F}) \in \mathcal{D}_{k}$ with $k=|F|$.

Proof: Let $\bar{F} \in \mathcal{Q}_{i}$ and $P_{\mid F}$ the associated suborder of $P$. We will show that $\psi(\bar{F}) \in \mathcal{E}$. By construction, we have $\sum_{t=1}^{l} x_{t} \leq m-1$, so we have $x_{0}=m-1-\sum_{t=1}^{l} x_{t} \in \mathbb{N}$.

We have $\sum_{t=0}^{l} t \cdot x_{t}=|F|$ and, as $F \in \tilde{\mathcal{A}}_{i}, 0 \leq|F| \leq n-l$. Thus, $\psi(\bar{F}) \in D_{k}$ with $k=\sum_{t=0}^{l} t \cdot x_{t}$.

Now we will show that $\psi$ is a bijection.

$\psi$ is injective: Suppose $\psi\left(\overline{F_{1}}\right)=\psi\left(\overline{F_{2}}\right)$ then $P_{\mid F_{1}}$ is isomorphic to $P_{\mid F_{2}}$ hence $\overline{F_{1}}=\overline{F_{2}}$.

$\psi$ is surjective: Let $x=\left(x_{0}, \ldots, x_{l}\right) \in \mathcal{D}_{k}$, i.e. $\sum_{t=0}^{l} x_{t}=m-1$ and $k=\sum_{t=0}^{l} t \cdot x_{t}$. For each $j \in[1, l]$, take $x_{j}$ chains of size $j$. Define the poset $P_{\mathcal{X}}$ as the parallel sum of all the 
defined chains. Since the number of chains in $P_{\mathcal{X}}$ is at most $m-1$ and the size of each chain is bounded by $l$, then $P_{\mathcal{X}}$ is isomorphic to a suborder $P_{\mid F}$ of $P$ with $F \cap C(i)=\emptyset$. Thus $\psi(\bar{F})=x$.

Example 2. (Example 1 continued) For $i=5$, as $5 \in C_{3}$, the elements of $\tilde{\mathcal{A}}_{5}$ are the feasible coalitions $F \in \mathcal{F}_{\Sigma}$ such that $F \cap C_{3}=\emptyset$, that is, $5 \notin F$ and $6 \notin F$ :

$$
\tilde{\mathcal{A}}_{5}=\{\emptyset,\{1\},\{3\},\{1,3\},\{1,2\},\{1,2,3\},\{3,4\},\{1,3,4\},\{1,2,3,4\}\} ;
$$

The size of an element of $\tilde{\mathcal{A}}_{5}$ is between 0 and $n-l=6-2=4$. The sets $\mathcal{D}_{k}$ for $k \in[0,4]$ are:

$$
\begin{gathered}
\mathcal{D}_{0}=\{(2,0,0)\}, \quad \mathcal{D}_{1}=\{(1,1,0)\}, \quad \mathcal{D}_{2}=\{(0,2,0),(1,0,1)\}, \\
\mathcal{D}_{3}=\{(0,1,1)\}, \quad \mathcal{D}_{4}=\{(0,0,2)\} .
\end{gathered}
$$

And:

$$
\mathcal{E}=\bigcup_{k=0}^{4} \mathcal{D}_{k}=\{(2,0,0),(1,1,0),(0,2,0),(1,0,1),(0,1,1),(0,0,2)\}
$$

The set $\mathcal{D}_{2}$ contains two codes which correspond to two equivalence classes modulo $\mathcal{R}_{5}$. These two classes contain coalitions of size $k=2$. The class associated with the code $(0,2,0)$ contains feasible coalitions that are built by taking exactly one player from two distinct chains not containing the player 5 . The only such coalition is $\{1,3\}$. The class associated with the code $(1,0,1)$ contains feasible coalitions that are built by discarding one chain (the 1 in the first position), never taking only one player from a chain (the zero in the second position), and taking two players in a given chain, that doesn't contain the player 5 . The feasible coalitions that satisfy this pattern are $\{1,2\}$ and $\{3,4\}$. Indeed we have $\{1,2\} \mathcal{R}_{5}\{3,4\}$. We can see also that $\{1,2,3\} \mathcal{R}_{5}\{1,4,3\}$ and the two coalitions share the code $(0,1,1) \in \mathcal{D}_{3}$.

Let $x \in \mathcal{E}$ and denote by $\mathcal{A}_{i}^{x}$ the equivalence class $\psi^{-1}(x)$, where $\psi$ is the bijection defined in proposition 6. By Proposition 5, we have $\left|F \backslash F^{\prime}\right|=1$ for all $\left(F, F^{\prime}\right) \in \mathcal{A}_{i}$. Thus, equation (2) can be rewritten as:

$\varphi_{i}(v)=\frac{1}{C h \downarrow(N)} \sum_{k=0}^{n-l} \sum_{x \in \mathcal{D}_{k}} \sum_{F \in \mathcal{A}_{i}^{x}} C h^{\downarrow}\left(F \cup\{i\}^{\Sigma} \backslash\{i\}\right) \cdot C h^{\uparrow}\left(F \cup\{i\}^{\Sigma}\right) \cdot\left(v\left(F \cup\{i\}^{\Sigma}\right)-v\left(F \cup\{i\}^{\Sigma} \backslash\{i\}\right)\right)$.

The following lemma states that $C h^{\downarrow}\left(F \cup\{i\}^{\Sigma} \backslash\{i\}\right) \cdot C h^{\uparrow}\left(F \cup\{i\}^{\Sigma}\right)$ depends only on the class of $F$. 
Lemma 2. Let all the chains in $P$ have the same length and $x \in \mathcal{E}$. Then for all $F_{1}, F_{2} \in$ $\mathcal{A}_{i}^{x}$, we have:

$$
C h^{\downarrow}\left(F_{1} \cup\{i\}^{\Sigma} \backslash\{i\}\right) \cdot C h^{\uparrow}\left(F_{1} \cup\{i\}^{\Sigma}\right)=C h^{\downarrow}\left(F_{2} \cup\{i\}^{\Sigma} \backslash\{i\}\right) \cdot C h^{\uparrow}\left(F_{2} \cup\{i\}^{\Sigma}\right) .
$$

\section{Proof:}

Let $x \in \mathcal{E}$ and $F_{1}, F_{2} \in \mathcal{A}_{i}^{x}$. Recall that for any $F \in \mathcal{F}_{\Sigma}, C h^{\downarrow}(F)$ is the number of linear extensions of $P_{\mid F}$ and $C h^{\uparrow}(F)$ is the number of linear extensions of $P_{\mid N \backslash F}$.

Since $P_{\mid F_{1}}$ is isomorphic to $P_{\mid F_{2}}$, then $P_{\mid F_{1} \cup\{i\}^{\Sigma} \backslash\{i\}}$ is isomorphic to $P_{\mid F_{2} \cup\{i\}^{\Sigma} \backslash\{i\}}$. Therefore, we have:

$C h^{\downarrow}\left(F_{1} \cup\{i\}^{\Sigma} \backslash\{i\}\right)=C h^{\downarrow}\left(F_{2} \cup\{i\}^{\Sigma} \backslash\{i\}\right)$.

On the other hand, since all the chains are of the same length, then $P_{\mid N \backslash\left[F_{1} \cup\{i\}^{\Sigma} \backslash\{i\}\right]}$ is isomorphic to $P_{\mid N \backslash\left[F_{2} \cup\{i\}^{\Sigma} \backslash\{i\}\right]}$. Therefore, we also have:

$$
C h^{\uparrow}\left(F_{1} \cup\{i\}^{\Sigma} \backslash\{i\}\right)=C h^{\uparrow}\left(F_{2} \cup\{i\}^{\Sigma} \backslash\{i\}\right) .
$$

Hence, we have:

$$
C h^{\downarrow}\left(F_{1} \cup\{i\}^{\Sigma} \backslash\{i\}\right) \cdot C h^{\uparrow}\left(F_{1} \cup\{i\}^{\Sigma}\right)=C h^{\downarrow}\left(F_{2} \cup\{i\}^{\Sigma} \backslash\{i\}\right) \cdot C h^{\uparrow}\left(F_{2} \cup\{i\}^{\Sigma}\right) .
$$

Thus, we can associate with each class $\mathcal{A}_{i}^{x}$ the nonnegative integer

$$
\alpha_{x}=C h^{\downarrow}\left(F \cup\{i\}^{\Sigma} \backslash\{i\}\right) \cdot C h^{\uparrow}\left(F \cup\{i\}^{\Sigma}\right)
$$

with $F$ being any coalition in $\mathcal{A}_{i}^{x}$. The following lemma gives a formula for $\alpha_{x}$.

Lemma 3. Let $x \in \mathcal{E}$ and $k=\sum_{t=0}^{l} t \cdot x_{t}$. We have

$$
\alpha_{x}=\frac{(k+h(i)) ! \cdot(n-k-h(i)-1) !}{h(i) ! \cdot(l-h(i)-1) ! \cdot \prod_{t=0}^{l}[t ! \cdot(l-t) !]^{x_{t}}} .
$$

Proof: Let $x \in \mathcal{E}$ with $k=\sum_{t=0}^{l} t \cdot x_{t}$ and $F \in \mathcal{A}_{i}^{x}$. Let $F^{\prime}=F \cup\{i\}^{\Sigma} \backslash\{i\}$ and $p_{j}=\left|F^{\prime} \cap C_{j}\right|$, $j=1, \ldots m$, where $C_{j}$ is a chain of $P$.

$C h^{\downarrow}\left(F^{\prime}\right)$ is the number of linear extensions of $P_{\mid F^{\prime}}$ which is the parallel sum of disjoint chains of sizes $p_{j} j=1, \ldots m$. Thus, we have [22]: $C h^{\downarrow}\left(F^{\prime}\right)=\frac{\left|F^{\prime}\right| !}{\prod_{j=1}^{m} p_{j} !}$. 
In the same way, we obtain: $\left.C h^{\uparrow}\left(F \cup\{i\}^{\Sigma}\right)\right)=C h^{\uparrow}\left(F^{\prime} \cup\{i\}\right)=\frac{\left(n-\left|F^{\prime}\right|-1\right) ! \cdot(l-h(i))}{\prod_{j=1}^{m}\left(l-p_{j}\right) !}$.

It follows that $C h^{\downarrow}\left(F^{\prime}\right) \cdot C h^{\uparrow}\left(F^{\prime} \cup\{i\}\right)=\frac{\left|F^{\prime}\right| ! \cdot\left(n-\left|F^{\prime}\right|-1\right) ! \cdot(l-h(i))}{\prod_{j=1}^{m} p_{j} ! \cdot\left(l-p_{j}\right) !}$.

For $t=0,1 \ldots, l, t \neq h(i)$, the number of chains $C_{j}$ such that $p_{j}=t$ is $x_{t}$. The number of chains $C_{j}$ such that $p_{j}=h(i)$ is $x_{h(i)}+1$. Thus,

$$
C h^{\downarrow}\left(F^{\prime}\right) \cdot C h^{\uparrow}\left(F^{\prime} \cup\{i\}=\frac{\left|F^{\prime}\right| ! \cdot\left(n-\left|F^{\prime}\right|-1\right) ! \cdot(l-h(i))}{\prod_{t=0}^{l}[t ! \cdot(l-t) !]^{x_{t}} \cdot h(i) ! \cdot(l-h(i)) !} .\right.
$$

Finally, observing that $\left|F^{\prime}\right|=k+h(i)$, we get: $\alpha_{x}=\frac{(k+h(i)) ! \cdot(n-k-h(i)-1) !}{\prod_{t=0}^{l}[t ! \cdot(l-t) !]^{x} t \cdot h(i) ! \cdot(l-h(i)-1) !}$.

Now we can rewrite equation (5) using $\alpha_{x}$ as follows:

$$
\varphi_{i}(v)=\frac{1}{C h^{\downarrow}(N)} \sum_{k=0}^{n-l} \sum_{x \in \mathcal{D}_{k}} \alpha_{x} \cdot \sum_{F \in \mathcal{A}_{i}^{x}}\left(v\left(F \cup\{i\}^{\Sigma}\right)-v\left(F \cup\{i\}^{\Sigma} \backslash\{i\}\right)\right) .
$$

In order to compute $\varphi_{i}(v)$ using (6), we need to enumerate, for $k=0, \ldots, n-l$, all the elements of $\mathcal{D}_{k}$. Now, we describe a backtracking algorithm to enumerate all the encodings $x=\left(x_{0}, \ldots, x_{l}\right) \in \mathbb{N}^{l+1}$ in $\mathcal{D}_{k}$. At a depth $j$, we suppose that all the entries $x_{l}, \ldots, x_{j-1}$ have already been filled. The remaining values $m^{\prime}=m-1-\sum_{t=j-1}^{l} x_{t}$ and $k^{\prime}=k-\sum_{t=j-1}^{l} t \cdot x_{t}$ will be decomposed on $x_{0}, \ldots, x_{j}$ if $k^{\prime} \leq j . m^{\prime}$, otherwise there is no possible completion of the vector $x$. Whenever $k^{\prime}=0$ the only solution is to put all the remaining chains in $x_{0}$. The first call to Algorithm 4 is $\operatorname{Gen-\operatorname {Class}}(l, k, m-1)$ with $0 \leq k \leq l .(m-1)$. 


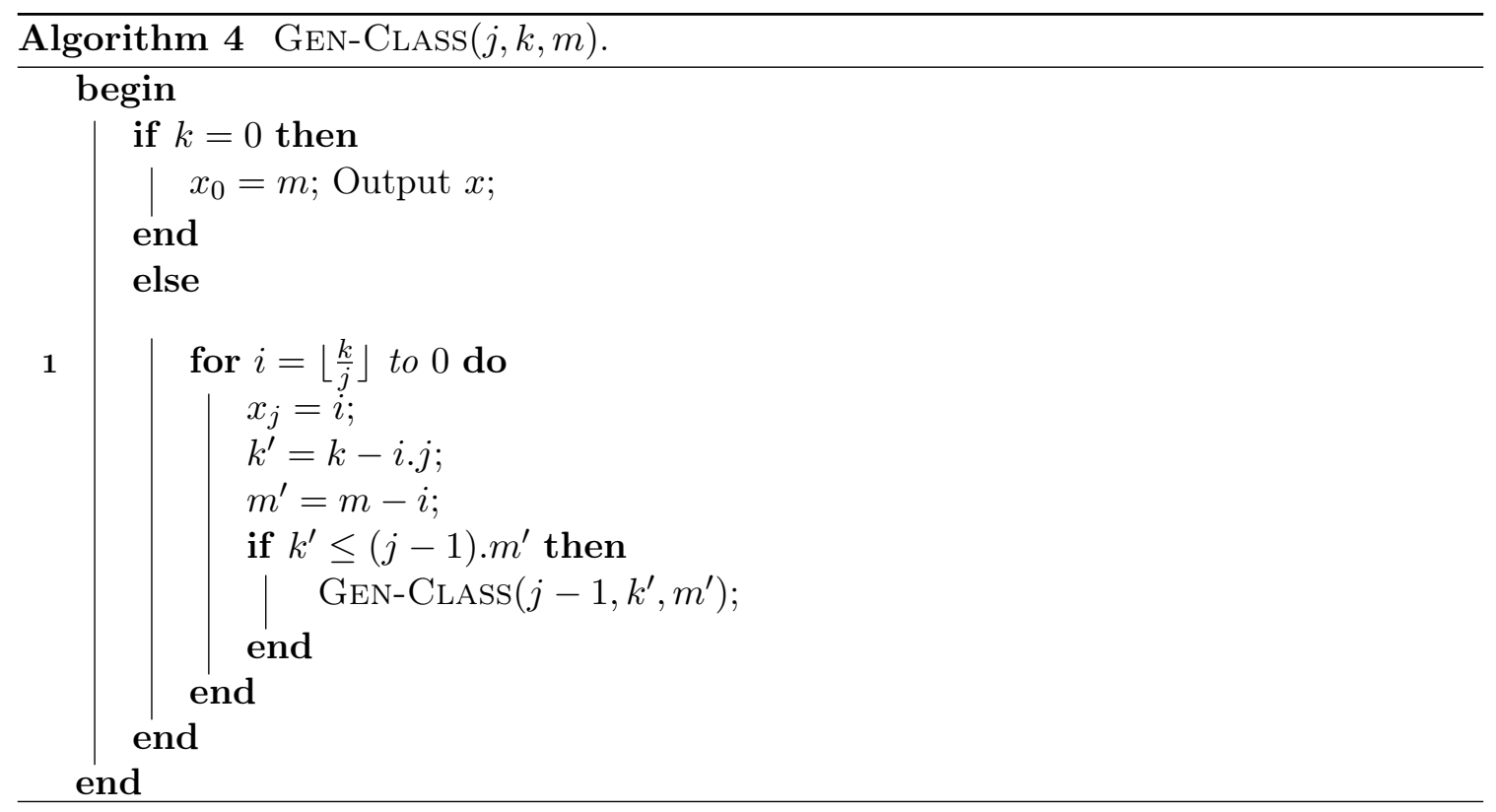

Proposition 7. Let $k$ be an integer in $[0, n-l]$. Algorithm 4 generates all the vectors in $\mathcal{D}_{k}$ in $O\left(k . l .\left|\mathcal{D}_{k}\right|\right)$ using polynomial space.

Proof: First, we will show by induction on the integer $j \in[1, l]$, that all the vectors $x=\left(x_{0}, \ldots, x_{l}\right)$ generated by $\operatorname{Gen-CLASs}(j, k, m-1)$ satisfy $\sum_{t=0}^{j} t x_{t}=k$ and $\sum_{t=0}^{j} x_{t}=m-1$ and therefore belong to $\mathcal{D}_{k}$.

For $j=1$, the algorithm generates a unique vector $x$ with $x_{0}=m-1-k$ and $x_{1}=k$ and all other entires are null, which satisfies $\sum_{t=0}^{j} t x_{t}=k$ and $\sum_{t=0}^{j} x_{t}=m-1$.

Suppose now that every vector $x$, generated by $\operatorname{Gen-CLASS}\left(j^{\prime}, k^{\prime}, m^{\prime}\right)$, where $1 \leq j^{\prime}<j$, is correct. The call GEn-ChASS $(j, k, m-1)$ will do the following affectations:

$$
x_{j}=i, \quad k^{\prime}=k-i j, \quad m^{\prime}=m-1-i
$$

and then call Gen-Chass $\left(j-1, k^{\prime}, m^{\prime}\right)$. By the induction hypothesis, any output $x$ of $\operatorname{Gen-CLass}\left(j-1, k^{\prime}, m^{\prime}\right)$ satisfies: $\sum_{t=0}^{j-1} t x_{t}=k^{\prime}$ and $\sum_{t=0}^{j-1} x_{t}=m^{\prime}$.

Thus, we have $\sum_{t=0}^{j} t x_{t}=k^{\prime}+j x_{j}=k-i j+j i=k$ and $\sum_{t=0}^{j} x_{t}=m^{\prime}+x_{j}=m-1-i+i=$ $m-1$. Thus, for all $j \in[1, l]$, the vectors $x$ generated by $\operatorname{Gen-Class}(j, k, m-1)$ satisfy $\sum_{t=0}^{j} t x_{t}=k$ and $\sum_{t=0}^{j} x_{t}=m-1$. For $j=l$, we obtain $\sum_{t=0}^{l} t x_{t}=k$ and $\sum_{t=0}^{l} x_{t}=m-1$ that is, $x \in \mathcal{D}_{k}$. 
Now, we will show that if $a=\left(a_{0}, \ldots, a_{l}\right) \in \mathcal{D}_{k}$, then $a$ is generated by Algorithm 4 with the input $(l, k, m-1)$. First, note that, the values of $x_{l}$ generated by the algorithm are the integers between 0 and $\left\lfloor\frac{k}{l}\right\rfloor$. Since $a \in D_{k}$, we have $k=\sum_{t=0}^{l} t \cdot a_{t}$ and $\frac{k}{l}=a_{l}+\frac{\sum_{t=0}^{l-1} t \cdot a_{t}}{l}$.

Therefore $a_{l} \leq\left\lfloor\frac{k}{l}\right\rfloor$ and the element $a_{l}$ will be generated by the algorithm. Next, suppose that the partial solution $\left(a_{j+1}, \ldots a_{l}\right)$ has already been generated. Then Algorithm 4 will be called with the arguments $\left(j, \sum_{t=0}^{j} t \cdot a_{t}, m-1-\sum_{t=j+1}^{l} a_{t}\right)$. The values $x_{j}$ generated by the algorithm are the integers between 0 and $\left\lfloor\frac{\sum_{t=0}^{j} t \cdot a_{t}}{j}\right\rfloor$. Since $a_{j} \leq\left\lfloor\frac{\sum_{t=0}^{j} t \cdot a_{t}}{j}\right\rfloor$, the partial solution $\left(a_{j}, a_{j+1}, \ldots a_{l}\right)$ will be generated. Once the sequence $\left(a_{1}, \ldots, a_{l}\right)$ generated, the algorithm will generate $a_{0}$ by $a_{0}=m-1-\sum_{t=1}^{l} a_{t}$. Thus the solution $a$ will be generated by the algorithm.

Each call of the algorithm will cost $O(k)$ (see Line 1), and the depth of the execution tree is bounded by $l$ the size of the vector. Thus the total cost of the algorithm is bounded by $O\left(k . l .\left|\mathcal{D}_{k}\right|\right)$, since each call has a leaf which is a solution.

The space used by the algorithm corresponds to a chain from the root to a leaf and is bounded by $O(l)$.

Proposition 8. We have $\left|\mathcal{D}_{k}\right| \in O\left(k^{l}\right)$.

Proof: The elements of $\mathcal{D}_{k}$ correspond to the leaves of the search tree of Algorithm 4. The depth of the tree is $l$ and its branching factor is bounded by $O(k)$. Thus $\left|\mathcal{D}_{k}\right| \in O\left(k^{l}\right)$.

Observe however, that even if we can enumerate efficiently all the elements of $\mathcal{D}_{k}$ for $k=0, \ldots n-l$, and we have a formula for each $\alpha_{x}$, computing the Shapley value using Equation (6) remains hard for general $v$. Indeed, if $v$ is given by an oracle, we must execute at least $\left|\mathcal{A}_{i}^{x}\right|$ calls to the oracle in order to compute $v\left(F \cup\{i\}^{\Sigma}\right)-v\left(F \cup\{i\}^{\Sigma} \backslash\{i\}\right)$ for each $F \in \mathcal{A}_{i}^{x}$. In the following, we will consider particular games on a product of chains for which the computation can be carried efficiently.

The model of weighted graph games captures the interactions between pairs of players. This is done by considering an undirected graph $G=(N, E)$ with an integer weight $v_{i j}$ for each edge $\{i, j\} \in E$. A positive $v_{i j}$ means that there are synergies between the two players, and a negative one that the presence of both players in a coalition will decrease its worth, because of, for instance, a conflict between $i$ and $j$. We define a cooperative game $(N, \Sigma, v)$ by:

$$
v(S)=\sum_{\{i, j\} \subseteq S} v_{i j} \quad \forall S \in \mathcal{F}_{\Sigma}
$$


When $\Sigma=\emptyset$, we recover the classical weighted graph games studied by Deng and Papadimitriou [8] who gave a simple formula for computing the Shapley value of these games. We will show that the computation of the Shapley value remains easy when the lattice of feasible coalitions is isomorphic to a product of chains with the same fixed length.

Proposition 9. Let $(N, \Sigma, v)$ be a weighted graph game and $i \in N$. We have,

$$
\varphi_{i}(v)=\frac{1}{C h^{\downarrow}(N)} \sum_{k=0}^{n-l} \sum_{x \in \mathcal{D}_{k}} \sum_{j \neq i} \beta_{i j}^{x} \cdot \alpha_{x} \cdot v_{i j}, \text { where } \beta_{i j}^{x}=\left|\left\{F \in \mathcal{A}_{i}^{x} \mid j \in F \cup\{i\}^{\Sigma}\right\}\right| .
$$

Proof: Let $i \in N$ and $F \in \mathcal{A}_{i}$. We have

$$
v\left(F \cup\{i\}^{\Sigma}\right)-v\left(F \cup\{i\}^{\Sigma} \backslash\{i\}\right)=\sum_{j \in F \cup\{i\}^{\Sigma} \backslash\{i\}} v_{i j}
$$

Thus, (6) becomes $\varphi_{i}(v)=\frac{1}{C h^{\downarrow}(N)} \sum_{k=0}^{n-l} \sum_{x \in \mathcal{D}_{k}} \sum_{F \in \mathcal{A}_{i}^{x}}\left(\alpha_{x} \cdot \sum_{j \in F \cup\{i\}^{\Sigma} \backslash\{i\}} v_{i j}\right)$, which can be rewritten as:

$$
\varphi_{i}(v)=\frac{1}{C h^{\downarrow}(N)} \sum_{k=0}^{n-l} \sum_{x \in \mathcal{D}_{k}} \sum_{j \neq i} \sum_{\substack{F \in \mathcal{A}_{i}^{x}, j \in F \cup\{i\}^{\Sigma}}} \alpha_{x} \cdot v_{i j}
$$

Finally, we obtain:

$$
\varphi_{i}(v)=\frac{1}{C h^{\downarrow}(N)} \sum_{k=0}^{n-l} \sum_{x \in \mathcal{D}_{k}} \sum_{j \neq i} \beta_{i j}^{x} \cdot \alpha_{x} \cdot v_{i j}
$$

Example 3. (Example 1 continued)

Let $i=5, j=1$ and $x=(0,1,1)$. We have $\mathcal{A}_{i}^{x}=\{\{1,2,3\},\{1,4,3\}\}$ and $\beta_{i j}^{x}=2$ since the two coalitions in $\mathcal{A}_{i}^{x}$ contain the player 1 .

The next proposition gives a formula for $\beta_{i j}^{x}$ :

Lemma 4. Let $i \neq j \in N$ and $x \in \mathcal{E}$. Then 


$$
\beta_{i j}^{x}=\left\{\begin{array}{l}
0, \quad \text { if } j \rightarrow i, \\
\frac{(m-1) !}{\prod_{t=0}^{l} x_{t} !}, \text { if } i \rightarrow j, \\
\frac{(m-2) !}{\prod_{t=0}^{l} x_{t} !} \cdot \sum_{t=h(j)+1}^{l} x_{t}, \text { otherwise. }
\end{array}\right.
$$

Proof: Recall that the number of partitions of a set $X$, with $|X|=n$ to parts $X_{0}, \ldots, X_{l}$, such that $\left|X_{t}\right|=x_{t}$, with $\sum_{t=0}^{l} x_{t}=n$ is given by the multinomial coefficient [7, p. 27]:

$$
\left(\begin{array}{c}
n \\
x_{0}, \ldots, x_{l}
\end{array}\right)=\frac{n !}{\prod_{t=0}^{l} x_{t} !}
$$

We will distinguish 3 cases according to whether $i$ and $j$ are comparable in $P$ or not.

1. $j \rightarrow i$, i.e. $i$ is below $j$ in a chain. Then, $j \notin F \cup\{i\}^{\Sigma}$ for any $F \in \mathcal{A}_{i}^{x}$, hence $\beta_{i j}^{x}=0$.

2. $i \rightarrow j$, i.e. $j$ is below $i$ in a chain. Then, $j \in F \cup\{i\}^{\Sigma}$ for all $F \in \mathcal{A}_{i}^{x}$. Hence, we have:

$$
\beta_{i j}^{x}=\left|\mathcal{A}_{i}^{x}\right|=\left(\begin{array}{c}
m-1 \\
x_{0}, \ldots, x_{l}
\end{array}\right)=\frac{(m-1) !}{\prod_{t=0}^{l} x_{t} !} .
$$

3. $i \nrightarrow j$ and $j \nrightarrow i$, i.e. $i$ and $j$ are not in the same chain. Let $\theta_{j}$ be the height of the maximal element of $F \in \mathcal{A}_{i}^{x}$ which is in the same chain as $j$. For a given $\theta_{j}$, the choice of $F$ amounts at partitioning the $m-2$ chains not containing neither $i$ nor $j$, to sets $X_{0}, \ldots, X_{l}$ with $\left|X_{t}\right|=x_{t}^{\prime}$, where

$$
x_{t}^{\prime}= \begin{cases}x_{t}-1 & \text { if } t=\theta_{j}+1 \\ x_{t} & \text { otherwise }\end{cases}
$$

Since $\theta_{j}$ can take all the integer values in the interval $[h(j), l-1]$, we have:

$$
\beta_{i j}^{x}=\sum_{h(j) \leq \theta_{j} \leq l-1}\left(\begin{array}{c}
m-2 \\
x_{0}^{\prime}, \ldots, x_{l}^{\prime}
\end{array}\right) \text {, where }\left(\begin{array}{c}
m-2 \\
x_{0}^{\prime}, \ldots, x_{l}^{\prime}
\end{array}\right)=\frac{(m-2) !}{\prod_{t=0}^{l} x_{t}^{\prime} !}=\frac{(m-2) ! \cdot x_{\theta_{j}+1}}{\prod_{t=0}^{l} x_{t} !} .
$$


Therefore, $\beta_{i j}^{x}=\sum_{h(j) \leq \theta_{j} \leq l-1} \frac{(m-2) ! \cdot x_{\theta_{j}+1}}{\prod_{t=0}^{l} x_{t} !}=\frac{(m-2) !}{\prod_{t=0}^{l} x_{t} !} \cdot \sum_{h(j) \leq \theta_{j} \leq l-1} x_{\theta_{j}+1}$

We can rewrite the last equation as: $\beta_{i j}^{x}=\frac{(m-2) !}{\prod_{t=0}^{l} x_{t} !} \cdot \sum_{t=h(j)+1}^{l} x_{t}$.

Theorem 1. The Shapley value $\varphi_{i}$ of a player $i$ in a weighted graph game on a product of $m$ chains with the same length $l-1$ can be computed in $O\left(n^{l+3}\right)$, where $n$ is the number of players. For fixed $l$, it can be computed in polynomial time.

\section{Proof:}

Let $P$ be the sum of $m$ disjoint chains of length $l-1$ and $i \in N$, where $N$ is the set of all the elements of the $m$ chains. Let $|N|=n$. From Proposition 9, the Shapley value can be computed using the formula:

$$
\varphi_{i}(v)=\frac{1}{C h^{\downarrow}(N)} \sum_{k=0}^{n-l} \sum_{x \in \mathcal{D}_{k}} \sum_{j \neq i} \beta_{i j}^{x} \cdot \alpha_{x} \cdot v_{i j}, \text { where } \beta_{i j}^{x}=\left|\left\{F \in \mathcal{A}_{i}^{x} \mid j \in F \cup\{i\}^{\Sigma}\right\}\right| .
$$

According to [22], the number of linear extensions $C h^{\downarrow}(N)$ of $P$ is $C h^{\downarrow}(N)=\frac{n !}{(l !)^{m}}$, thus $C h^{\downarrow}(N)$ can be computed in $O(n+m)=O(n)$ since $n \leq m$. From Proposition 7, the equivalence classes in $\mathcal{D}_{k}$ can be generated in $O\left(k . l .\left|\mathcal{D}_{k}\right|\right)$ for $k \in[0, n-1]$. Proposition 8 shows that the number of equivalence classes in $\mathcal{D}_{k}$ is bounded by $O\left(k^{l}\right)$. Using Lemma 3 and Lemma 4 , for each each equivalence class in $\mathcal{D}_{k}$ corresponding to $\mathcal{A}_{i}^{x}$, we compute both $\alpha_{x}$ and $\beta_{i j}^{x}$ in $O(n)$. Thus the total complexity is bounded by $O\left(n^{l+3}\right)$.

When $l$ is fixed, then $O\left(n^{l+3}\right)$ is a polynomial and therefore the total time complexity is bounded by a polynomial.

Remark 1. When Theorem 1 is restricted to classical graph games, i.e. $m=n$ and $l=1$, we obtain the complexity $O\left(n^{4}\right)$ which is larger than $O(n)$, the complexity obtained in [8] for each player. Indeed, for a classical graph game, the lattice is boolean and has several properties that cannot be exploited for the general case. The difficult in our case is the number of equivalence classes and how to compute them, whereas, for the boolean lattice, there are $n-1$ classes and this fact leads to a simplification of the formula.

Remark 2. If the chains are not of the same length, we define the relation $\mathcal{R}_{i}$ as follows:

$$
F_{1} \mathcal{R}_{i} F_{2} \Leftrightarrow\left(P_{\mid F_{1}} \cong P_{\mid F_{2}} \text { and } P_{\mid N \backslash F_{1}} \cong P_{\mid N \backslash F_{2}}\right) \text {. }
$$

Each class modulo $\mathcal{R}_{i}$ will be coded by two l-tuples of nonnegative integers, where $l$ is the maximal size of the chains. $C h^{\downarrow}\left(F \cup\{i\}^{\Sigma} \backslash\{i\}\right) \cdot C h^{\uparrow}\left(F \cup\{i\}^{\Sigma}\right)$ will be constant within each class and the number of classes corresponding to a cardinality $k$ of the class elements will be bounded by a polynomial in $k$ for constant $l$. 


\section{Concluding remarks}

We have addressed in this paper the issue of computing the Shapley value of a cooperative game on a lattice given by an implicational system. The structural properties of the lattice $\mathcal{L}_{\Sigma}$ of feasible coalitions play an important role in the design of efficient algorithms. Indeed, efficient algorithms for the computation of the Shapley value are likely to exist only on lattices for which the computation of the number of maximal chains traversing any element of the lattice can be done efficiently, since this computation is a prerequisite for the computation of the Shapley value. A lattice isomorphic to a product of chains is one such structure treated in this paper. There exist distributive lattices for which Nchains can be solved efficiently. For example, the lattices of ideals of series-parallel orders [22], or $N$-free orders with activity bounded by a constant [13]. An interesting issue to be addressed, would be whether the Shapley value can be computed efficiently for weighted graph games on these lattices.

When no efficient algorithm for computing the Shapley value is likely to exist, approximation becomes in order. When the lattice of feasible coalitions is distributive, approximating the number of maximal chains traversing an element of the lattice, amounts to approximate the number of linear extensions of a partial order. Methods have been proposed to deal with this issue, $[5,10,6]$. One may think also of reducing the number of coalitions considered in computing the Shapley value, following an idea used in [26] in searching for an optimal coalition structure. Of course, the quality of the approximation, will depend on the choice of the coalitions taken into consideration.

As presented in this paper, the model of cooperative games on implicational systems generalizes the notion of a conjunctive permission structure. We think that this model can be extended to involve disjunctive permission structures, and many other constraints on coalition formation, by allowing for implications with "negated" players. For example the implication $\overline{1} \overline{2} \overline{3} \rightarrow \overline{4}$ will mean that the absence of players 1,2 and 3 in a coalition will result in the absence of player 4 , and the implication $\overline{1} 2 \overline{3} \rightarrow 4$ will mean that the presence of player 2 in a coalition where the players 1 and 3 are absent, will entail the presence of player 4 .

Another issue is the study of the algorithmic aspects of other solution concepts for cooperative games on lattices such as the core.

Acknowledgment: The authors wish to thank anonymous referees for their suggestions which have greatly improved the presentation of the paper. This work has been funded by the CMEP Tassili 15MDU944-2015-2018 and the ANR project Graphen 2015-2019. The

first author has received a scholarship from the Algerian Ministry of Higher Education and Scientific Research. 


\section{References}

[1] Baixeries, J., Szathmary, L., Valtchev, P., and Godin, R. (2009). Yet a faster algorithm for building the hasse diagram of a concept lattice. In Formal Concept Analysis, 7th International Conference, ICFCA 2009, Darmstadt, Germany, May 21-24, 2009, Proceedings, pages $162-177$.

[2] Bertet, K. and Monjardet, B. (2010). The multiple facets of the canonical direct unit implicational basis. Theor. Comput. Sci., 411(22-24):2155-2166.

[3] Bilbao, J. M. and Edelman, P. H. (2000). The shapley value on convex geometries. Discrete Applied Mathematics, 103(1-3):33-40.

[4] Bordat, J.-P. (1986). Calcul pratique du treillis de galois d'une correspondance. Math. Sci. Hum., 96(5-6):31-47.

[5] Brightwell, G. and Winkler, P. (1991). Counting linear extensions. Order, 8(3):225-242.

[6] Bubley, R. and Dyer, M. (1999). Faster random generation of linear extensions. Discrete Mathematics, 201(1-3):81-88.

[7] Comtet, L. (1974). Advanced Combinatorics. The art of finite and infinite expansions. D. Reidel Publishing Company, P.O. Box 17, Dordrecht, Holland.

[8] Deng, X. and Papadimitriou, C. H. (1994). On the complexity of cooperative solution concepts. Mathematics of Operations Research, 19(2):257-266.

[9] Derks, J. and Gilles, R. (1995). Hierarchical organization structures and constraints on coalition formation. International Journal of Game Theory, 24(2):147-163.

[10] Ewacha, K., Rival, I., and Zaguia, N. (1997). Approximating the number of linear extensions. Theoretical Computer Science, 175(2):271-282.

[11] Faigle, U., Grabisch, M., Jiménez-Losada, A., and Ordóñez, M. (2016). Games on concept lattices: Shapley value and core. Discrete Applied Mathematics, 198:29-47.

[12] Faigle, U. and Kern, W. (1992). The shapley value for cooperative games under precedence constraints. International Journal of Game Theory, 21(3):249-266.

[13] Felsner, S. and Manneville, T. (2014). Linear extensions of N-free orders. Order, $32(2): 147-155$.

[14] Garg, V. K., Narahari, Y., and Narasimha Murty, M. (2013). Novel biobjective clustering (bigc) based on cooperative game theory. Knowledge and Data Engineering, IEEE Transactions on, 25(5):1070-1082. 
[15] Gilles, R. P., Owen, G., and Brink, R. (1992). Games with permission structures: The conjunctive approach. International Journal of Game Theory, 20(3):277-293.

[16] Grabisch, M. (2013). The core of games on ordered structures and graphs. Annals of Operations Research, 204(1):33-64.

[17] Hsiao, C.-R. and Raghavan, T. (1993). Shapley value for multichoice cooperative games, I. Games and Economic Behavior, 5(2):240-256.

[18] Kollias, K. and Roughgarden, T. (2011). Restoring pure equilibria to weighted congestion games. Automata, Languages and Programming: 38th International Colloquium, ICALP 2011, Zurich, Switzerland, July 4-8, 2011, Springer Berlin Heidelberg, pages $539-551$.

[19] Kuznetsov, S. O. and Obiedkov, S. A. (2001). Algorithms for the construction of concept lattices and their diagram graphs. In Principles of Data Mining and Knowledge Discovery, 5th European Conference, PKDD 2001, Freiburg, Germany, September 3-5, 2001, Proceedings, pages 289-300.

[20] Mannila, H. and Räihä, K.-J. (1992). On the complexity of inferring functional dependencies. Discrete Applied Mathematics, 40(2):237-243.

[21] Michalak, T. P., Aadithya, K. V., Szczepanski, P. L., Ravindran, B., and Jennings, N. R. (2013). Efficient computation of the shapley value for game-theoretic network centrality. Journal of Artificial Intelligence Research, 46:607-650.

[22] Möhring, R. H. (1989). Computationally tractable classes of ordered sets. In Algorithms and Order (Ivan Rival edition), Springer Netherlands, pages 105-193.

[23] Nourine, L. and Raynaud, O. (1999). A fast algorithm for building lattices. Inf. Process. Lett., 71(5-6):199-204.

[24] Nouweland, A., Tijs, S., Potters, J., and Zarzuelo, J. (1995). Cores and related solution concepts for multi-choice games. Zeitschrift für Operations Research, 41(3):289-311.

[25] Rudolph, S. (2017). Succinctness and tractability of closure operator representations. Theor. Comput. Sci., 658:327-345.

[26] Sandholm, T., Larson, K., Andersson, M., Shehory, O., and Tohmé, F. (1999). Coalition structure generation with worst case guarantees. Artificial Intelligence, 111(1-2):209 238.

[27] Shapley, L. S. (1953). A value for n-person games. In Kuhn, H. W. and Tucker, A. W., editors, Contributions to the Theory of Games (AM 28), volume II of Annals of Mathematics Studies., pages 307-317. Princeton University Press. 
[28] Stanley, R. P. (2011). Enumerative Combinatorics: Volume 1. Cambridge University Press, New York, NY, USA, 2nd edition.

[29] Wild, M. (1995). Computations with finite closure systems and implications. In Computing and Combinatorics, First Annual International Conference, COCOON '95, Xi'an, China, August 24-26, 1995, Proceedings, pages 111-120.

[30] Wild, M. (2017). The joy of implications, aka pure horn formulas: Mainly a survey. Theor. Comput. Sci., 658:264-292. 


\section{Appendix A. Computing the Shapley value using the Harsanyi dividends}

We explore the use of Harsanyi dividends and their recurrence formula in the computation of the Shapley value.

Following [12], we define for all $T \in \mathcal{F}_{\Sigma}, T \neq \emptyset$, the $T$-simple game $u_{T}: \mathcal{F}_{\Sigma} \rightarrow \mathbb{R}$ : by

$$
u_{T}(S)=\left\{\begin{array}{l}
1 \text { if } T \subseteq S \\
0 \text { otherwise }
\end{array}\right.
$$

When $\mathcal{F}_{\Sigma}=2^{N}, u_{T}$ is known as the $T$-unanimity game.

The family $\left\{u_{T}, T \in \mathcal{F}_{\Sigma} \backslash \emptyset\right\}$ is a basis of the vector space of all cooperative games defined on $\mathcal{F}_{\Sigma}$. Hence, each game $v: \mathcal{F}_{\Sigma} \rightarrow \mathbb{R}$ can be expressed uniquely as a linear combination of the games $u_{T}$ :

$$
v=\sum_{T \in \mathcal{F}_{\Sigma} \backslash \emptyset} \Delta_{T}^{v} \cdot u_{T}
$$

That is,

$$
\forall S \in \mathcal{F}_{\Sigma}, \quad v(S)=\sum_{T \in \mathcal{F}_{\Sigma} \backslash \emptyset} \Delta_{T}^{v} \cdot u_{T}(S)
$$

In view of the definition of the simple games $u_{T}$, the last equation becomes

$$
\forall S \in \mathcal{F}_{\Sigma}, \quad v(S)=\sum_{T \in \mathcal{F}_{\Sigma} \backslash \emptyset, T \subseteq S} \Delta_{T}^{v}
$$

The real coefficient $\Delta_{T}^{v}$ is called the Harsanyi dividend of the coalition $T$ in the game $v$.

Letting $\Delta_{\emptyset}^{v}=0$, Equation (A.3) yields the following recurrence formula for the dividends $\Delta_{T}^{v}, T \in \mathcal{F}_{\Sigma}$

$$
\Delta_{T}^{v}=\left\{\begin{array}{l}
0 \text { if } T=\emptyset, \\
v(T)-\sum_{S \in \mathcal{F}_{\Sigma}, S \subset T} \Delta_{S}^{v} \quad \text { otherwise. }
\end{array}\right.
$$

Using this recurrence formula to compute the dividends $\Delta_{T}^{v}$ is not efficient from the algorithmic point of view, since, to compute $\Delta_{T}^{v}$, we have to compute all the $\Delta_{S}^{v}$ 's, where $S \subset T$ is a feasible coalition.

We now turn to the evaluation of the Shapley value $\varphi_{i}\left(u_{T}\right)$ of the player $i \in N$ in the simple game $u_{T}$. Let us recall the definition of the hierarchical strength $h_{T}(i)$ of player $i$ in the feasible coalition $T$ [12]. A feasible ranking of the players in $N$ is a linear extension of the order $P$. $i \in T$ is $T$-maximal in the ranking $\pi$, if $i$ is maximal in the linear order 
induced by $\pi$ on $T$. Let $\mathcal{R}(N)$ be the set of all feasible rankings of $N$. The hierarchical strength $h_{T}(i)$ of player $i$ in the feasible coalition $T$ is then defined by:

$$
h_{T}(i)=\frac{1}{|\mathcal{R}(N)|} \cdot \mid\{\pi \in \mathcal{R}(N) \mid i \text { is } T \text {-maximal in } \pi\} \mid .
$$

Let $h_{T}=\sum_{i \in T} h_{T}(i)$. Then, the Shapley value of $i \in N$ in the game $u_{T}$ is given by [12]:

$$
\varphi_{i}\left(u_{T}\right)=\left\{\begin{array}{lr}
\frac{h_{T}(i)}{h_{T}}, & \text { if } i \in T \\
0, & \text { otherwise }
\end{array}\right.
$$

Using Lemma 4 in [12], which states that $h_{T}(i) \neq 0$ if and only if $i \in \max (T)$, we obtain:

$$
\varphi_{i}\left(u_{T}\right)= \begin{cases}\frac{h_{T}(i)}{h_{T}}, & \text { if } i \in \max (T) \\ 0, & \text { otherwise. }\end{cases}
$$

Then, by the linearity of the Shapley value and Equation (A.1), we have:

$$
\varphi_{i}(v)=\sum_{T \in \mathcal{F}_{\Sigma}, i \in \max (T)} \frac{h_{T}(i)}{h_{T}} \cdot \Delta_{T}^{v}
$$

In the case studied in Section $5,\left(\mathcal{F}_{\Sigma}, \subseteq\right)$ is the lattice of ideals of a partial order $P$. Then, an explicit expression of the dividend $\Delta_{T}^{v}$ can be obtained from Equation (A.3), using the Möbius inversion formula [28]:

$$
\Delta_{T}^{v}=\sum_{S \in \mathcal{F}_{\Sigma}} \mu(S, T) \cdot v(S)
$$

where $\mu$ is the Möbius function of the lattice $\left(\mathcal{F}_{\Sigma}, \subseteq\right)$. As $\left(\mathcal{F}_{\Sigma}, \subseteq\right)$ is the lattice of ideals of the partial order $P$, we know [28] that

$$
\mu(S, T)= \begin{cases}(-1)^{|T|-|S|}, & \text { if } S \subseteq T \text { and } T \backslash S \text { is an antichain of } P, \\ 0, & \text { otherwise. }\end{cases}
$$

The condition $S \subseteq T$ and $T \backslash S$ is an antichain of $P$ is equivalent to $S=T \backslash E$, where $E$ is a subset of $\max (T)$. We thus obtain the following expression for the dividend $\Delta_{T}^{v}$ :

$$
\Delta_{T}^{v}=\sum_{E \subseteq \max (T)}(-1)^{|E|} \cdot v(T \backslash E) .
$$


The Shapley value of player $i \varphi_{i}(v)$, given by Equation (A.5), becomes then

$$
\varphi_{i}(v)=\sum_{T \in \mathcal{F}_{\Sigma}, i \in \max (T)} \frac{h_{T}(i)}{h_{T}} \cdot \sum_{E \subseteq \max (T)}(-1)^{|E|} \cdot v(T \backslash E) .
$$

Again, the direct use of Equation (A.8) to compute the Shapley value is not efficient from the computational point of view. Indeed, we have to generate all the coalitions $T \in \mathcal{F}_{\Sigma}$ with $i \in \max (T)$ and all the subsets $E$ of $\max T$. 